\title{
ON THE NATURE OF PRESSURE-INDUCED COORDINATION CHANGES IN SILICATE MELTS AND GLASSES
}

\author{
Edward M. Stolper and Thomas J. Ahrens
}

Division of Geological and Planetary Sciences, California Institute of Technology

\begin{abstract}
Progressive decreases in the $\mathrm{Si}-\mathrm{O}-\mathrm{Si}$ angles between corner-shared silicate tetrahedra in glasses and melts with increasing pressure can lead to arrangements of oxygen atoms that can be described in terms of edge- or face-shared $\propto$ ctahedra. This mechanism of compression can account for the gradual, continuous increases in melt and glass densities from values at low pressure that indicate dominantly tetrahedral coordination of Si to values at several tens of GPa that suggest higher coordination. It also can explain the unquenchable nature of octahedrally coordinated $\mathrm{Si}$ in glasses, the absence of spectroscopically detectable octahedrally coordinated $\mathrm{Si}$ in glasses until they are highly compressed, the gradual and reversible transformation from tetrahedral to octahedral coordination in glasses once the transformation is detectable spectroscopically, and the fact that this transformation takes place in glass at room temperature. It may also have relevance to pressure-induced transformations from crystalline to glassy phases, the difficulty in retrieving some metastable high pressure crystalline phases at low pressure, and the observed differences between the pressures required for phase transformations in shock wave experiments on glasses and crystals.
\end{abstract}

\section{Introduction}

It is generally accepted that silicon is tetrahedrally coordinated by oxygen ions in silicate melts and glasses at $1 \mathrm{~atm}$ total pressure and that adjacent silicate tetrahedra form polymers by comer-sharing [e.g., Mozzi and Warren, 1969; Taylor and Brown, 1979; Okuno and Marumo, 1982]. By analogy with the structures of crystalline silicates at pressures of several tens of GPa and based on shock wave studies of the Hugoniot states of molten silicates [Rigden et al., 1984, 1987, and in preparation], it is likely that silicon is more highly coordinated by oxygens at high pressures. In this note we propose a mechanism by which the coordination of $\mathrm{Si}$ (and other cations found in tetrahedral coordination at low pressures) may increase in glasses and melts with increasing pressure.

Several observations constrain possible mechanisms of compression in silicate glasses and melts: (1) Shock wave studies of molten silicates in the system anorthite-diopside demonstrate that the compression from several tenths to several tens of GPa is gradual and continuous without any abrupt density changes [Rigden et al., 1984, 1987, and in preparation] and suggest that the mechanisms of compression may be continuous over this pressure range. It is widely believed [e.g., Navrotsky et al., 1985; Ohtani et al., 1985; Hemley et al., 1986] that the principal mechanism of compression in amorphous silicates at low pressures is increased packing efficiency of linked aluminosilicate tetrahedra by decreases in the ( $\mathrm{Si}, \mathrm{Al})-\mathrm{O}-(\mathrm{Si}, \mathrm{Al})$ angles between adjacent tetrahedra. (2) Glasses (or melts) held at elevated pressures and then brought rapidly to room temperature and pressure ("pressure-quenched") and examined by infrared or Raman spectroscopy show no features attributed to octahedrally coordinated silicon [Amdt and Stơffler, 1969; Arndt, 1983; Grimsditch, 1984; Hemley et al., 1986; Williams and Jeanloz, 1986]. This is even true for "permanently" densified glasses, which are presumed to have preserved on quenching part of the structural changes they experienced at high pressures. Albitic glasses quenched from high temperatures and pressures greater than $6 \mathrm{GPa}$ do have features in their NMR spectra that have been assigned to octahedrally coordinated aluminum [Ohtani et al., 1985]. (3) When infrared spectra of anorthitic glass are obtained in situ at high pressure and room temperature, the following is observed [Williams and Jeanloz, 1987]: Up to $20 \mathrm{GPa}$, only tetrahedrally coordinated silicon is observed. With increasing pressure, bands attributed to octahedrally coordinated silicon grow, while those attributed to tetrahedrally coordinated silicon decrease. This transformation is continuous and reversible. After pressure-quenching to $1 \mathrm{~atm}$ from conditions under which octahedrally coordinated silicon was observed, only bands attributed to tetrahedrally coordinated silicon were observed.

\section{The Proposal}

What must be sought is a mechanism of compression that (1) begins with only tetrahedral coordination at low pressure and leads continuously to octahedral coordination at high pressure; (2) does not generate spectroscopically observable octahedrally coordinated silicon until after substantial compression; (3) produces, with increasing pressure, a continuous transformation of tetrahedral to octahedral coordination as detected by infrared spectroscopy at pressures above the first appearance of octahedrally coordinated silicon; (4) proceeds at room temperature; and $(5)$ is reversible and usually unquenchable.

Figure 1 illustrates the kind of mechanism we propose can satisfy these constraints. A chain of silicate tetrahedra with an $\mathrm{Si}-\mathrm{O}-\mathrm{Si}$ angle of $180^{\circ}$ (Figure 1a) is compressed by decreasing this angle. Figures 1a-1e show various stages in this continuous compression. A comparison of Figures le and 1f, both of which have the same placement of oxygen ions ( $\mathrm{Si}-\mathrm{O}-\mathrm{Si}=100^{\circ}$ in Figure le) illustrates our basic point: This configuration of oxygen atoms can be viewed either as a highly distorted and compressed chain of tetrahedra or as a chain of face-shared octahedra. Figure 1 thus illustrates a possible mechanism by which a low pressure, low density arrangement of oxygen tetrahedra can transform continuously into a closely packed arrangement of oxygen octahedra. Although we have not illustrated it, the chain of tetrahedra shown in Figure 1a could also have been compressed into a chain of edge-shared pentahedra.

Although the end product of compression of the chain of tetrahedra shown in Figure 1a is a chain of face-shared octahedra (which based on Pauling's rules is not expected to be an energetically favorable configuration), edge-shared octahedral arrangements can also be produced by distortion and compression of groups of comer-shared tetrahedra. Figure 2a shows two highly distorted, linked rings of silicate tetrahedra; Figure $2 b$ shows that the central portion of this configuration of oxygen atoms can also be viewed as two edge-shared octahedra. Both Figures $2 a$ and $2 b$ are actually portions of the crystal structure of stishovite; the arrangement of oxygen atoms in crystalline stishovite, which is usually viewed as linked chains of edge-shared octahedra, could also be viewed as a network of irregular, corner-shared tetrahedra.

In transforming from Figure $1 \mathrm{e}$ to $1 \mathrm{f}$ or $2 \mathrm{a}$ to $2 \mathrm{~b}$, there is no movement of oxygen atoms. There will, however, be minor movement of $\mathrm{Si}$ atoms. In the pictures showing tetrahedral coordination, each $\mathrm{Si}$ is only chemically bonded to four oxygen atoms, although two other oxygens are nearby. If the Si bonds to these two additional oxygens, it would be expected to move across one of the faces of the oxygen tetrahedron so as to be more centrally located within the octahedron defined by the six nearest oxygen atoms.

Our proposal that the transformation of $\mathrm{Si}$ from tetrahedral to octahedral coordination in melts and glasses with increasing pressure is 


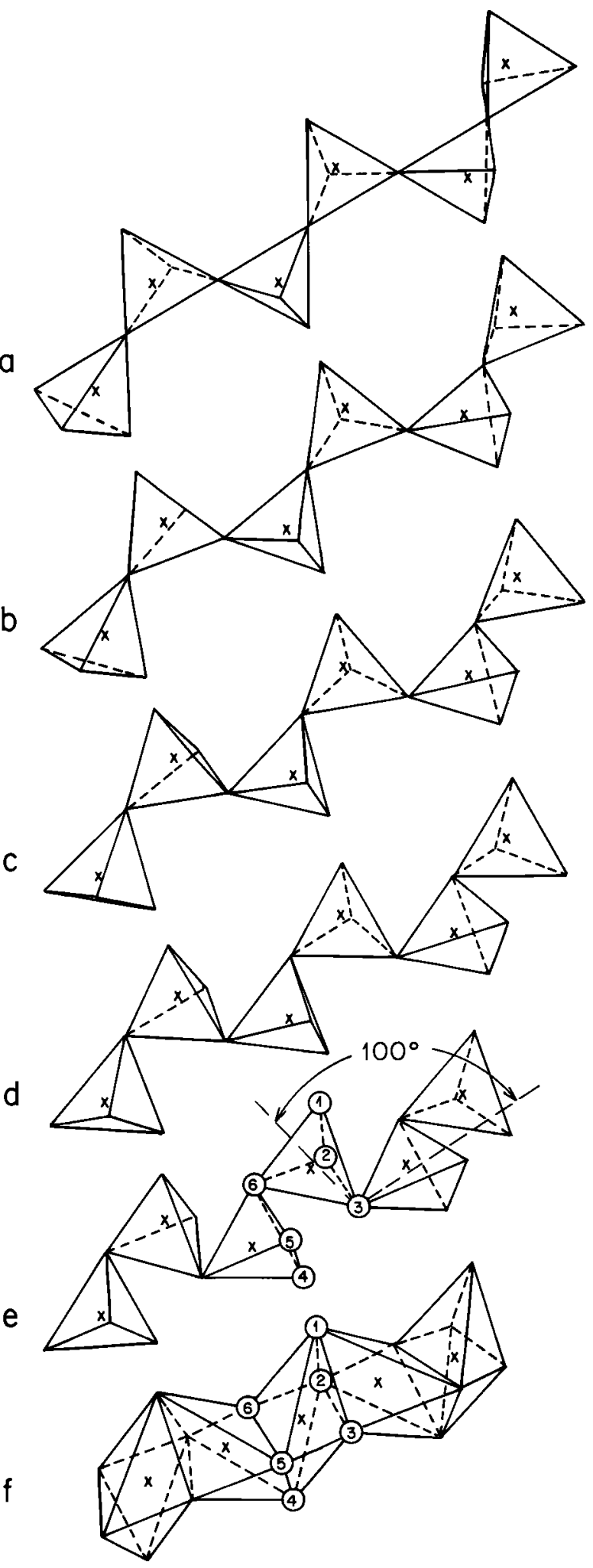

Fig. 1. Progressive distortion of a chain of $\mathrm{SiO}_{4}$ tetrahedra. Dashed lines are "hidden" edges of polyhedra. (a) Si-O-Si angle $=180^{\circ}$. (b) $\mathrm{Si}-\mathrm{O}-\mathrm{Si}$ angle $=160^{\circ}$. (c) Si-O-Si angle $=140^{\circ}$. (d) Si-O-Si angle $=120^{\circ}$. (e) Si-O-Si angle $=100^{\circ}$. (f) Same as chain in (e), except coordination polyhedra redrawn as a chain of face-shared octahedra. The numbered circles show the positions of the same oxygen atoms in (e) and (f). Si positions (crosses) have been moved to be near the centers of the octahedra in (f). Because of the finite length of the chain in (e), one Si and one $O$ (at lower left) are not reproduced in ( $f$ ).
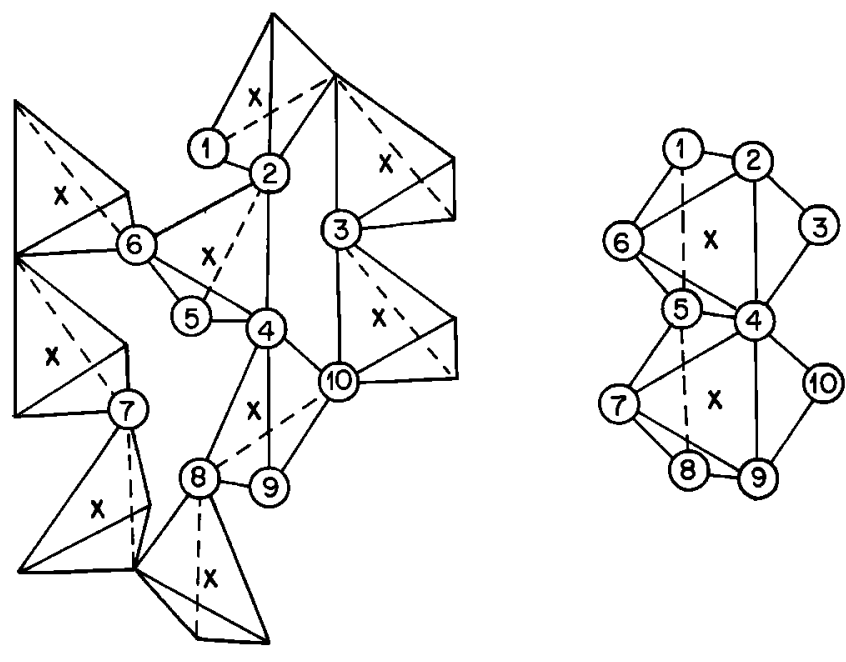

Fig. 2. Two views of a part of the structure of stishovite (modified from Bloss [1971, Figure 9-22]). (a) As distorted rings of distorted tetrahedra of oxygen atoms, each containing one $\mathrm{Si}$ atom. (b) As edge-shared, regular octahedra of oxygen atoms, each containing one $\mathrm{Si}$ atom. Dashed lines are the "hidden" edges of polyhedra. The numbered circles show the positions of the same oxygen atoms in (a) and (b). The crosses show $\mathrm{Si}$ atoms; the positions are shifted from (a) to (b). The oxygen packing of stishovite can be built up either from a distorted network of rings of corner-shared tetrahedra or from chains of regular, edge-shared octahedra. The configuration of ring sizes shown here (i.e., 5- and 6-membered rings) is not unique; the stishovite structure could also be built up of a range of smaller to larger ring sizes.

due to a continuous distortion of polymers constructed of corner-sharing tetrahedra rationalizes available data in the following ways:

(1) The gradual, continuous compression of amorphous silicates from low to high pressure without abrupt density changes is readily accounted for by this model. In particular, the mechanism of compression by decreasing Si-O-Si angles between adjacent tetrahedra can remain the same all the way from $1 \mathrm{~atm}$ to several tens of GPa. Once the configuration has reached one compatible with close, octahedral packing of oxygen atoms, the melt or glass will be expected to stiffen considerably. This has been observed in shock studies [Wackerle, 1962; Boslough et al, 1986; Rigden et al., 1987]. The proposed mechanism of compression may also help to explain why under shock compression the density of silica glass increases smoothly and approaches that of stishovite at lower pressure than does the density of shock compressed crystalline quartz [Schmitt, 1987], because the glass, unconstrained by crystal structure, can more readily distort into the proposed high density arrangement of oxygen polyhedra.

(2) It is not clear that when a packing of oxygens consistent with octahedral coordination of $\mathrm{Si}$ has been achieved, the material will necessarily give up its tetrahedral bonding environment. Thus, for example, it is conceivable that a glass could remain as a tetrahedral network (e.g., Figure 2a) to very high distortions and compressions. Such tetrahedra, however, would be highly distorted, and we think it likely that at some point, the $\mathrm{Si}$ atoms would begin to bond with the oxygen atoms that are being pressed in on them and a changeover to octahedral bonding (e.g., Figure $2 b$ ) would take place. Given the likely inhomogeneous nature of compression in real systems (i.e., a chain such as that shown in Figure 1 would probably compress irregularly), we would expect the change in bonding environment to take place at different pressures at different positions in the glass or melt structure. This can explain the range of pressures over which both octahedrally and tetrahedrally coordinated $\mathrm{Si}$ are observed in anorthitic glass [Williams 
and Jeanloz, 1987]. It is clear, however, that in the early stages of compression (e.g., Figures 1b, 1c) nothing but tetrahedral units are present in the glass or melt. This would explain why vibrational spectra of dense glasses, whether obtained at high pressure or at low pressure on permanentaly densified glasses, have revealed only tetrahedrally coordinated silicon.

(3) To first order, we would expect the compression of glasses and melts by the proposed mechanism to be elastic; that is, when we squeeze on the chain in Figure 1a, the Si-O-Si angle decreases, and when we release the pressure, it will spring back. In the range of compression over which the bonding environments change from tetrahedral to octahedral, we would still expect the transformation to be nearly reversible. We thus rationalize the observation that the transformation from tetrahedral to octahedral coordination of $\mathrm{Si}$ in anorthitic glass is reversible [Williams and Jeanloz, 1987]. In addition, when viewed in this light, it is not surprising that the transformation from low to high pressure packing of oxygens appears to be so difficult to pressurequench. This may also explain why octahedrally coordinated Al, whose presence has been inferred in melts at rather low pressures [e.g., Boettcher et al., 1984], has so rarely been observed in glasses pressurequenched to $1 \mathrm{~atm}$.

We note that in reality, compression of a glass or melt to several tens of GPa probably would not be entirely displacive, even if the mechanism that we have proposed dominates. In Figure 1, for example, the chain of tetrahedra may be bonded through metal cations to other silicate polymers, and these bonds would probably have to break as the silicate chain compresses and may not readily reform on release of pressure. Likewise, although it is possible to describe a network of octahedral chains in terms of distorted rings of tetrahedra (Figure 2), a given low pressure starting configuration of the silicate tetrahedra may not necessarily map directly into a three-dimensional network of octahedra. Thus, some bond breaking and diffusion would probably accompany extreme compression of complex melts and glasses. It is in this context that we would explain the permanent densification of glasses and hysteresis effects, though we still anticipate that most of the octahedrally coordinated Si would spring apart to the low pressure tetrahedrally coordinated configuration.

(4) It is important to emphasize that the proposed change of oxygen packing from the expanded low pressure tetrahedral network to a collapsed configuration involving octahedral packing geometries would not be limited by diffusion. The collapse of the structure and change in oxygen packing would take place essentially continuously and except for a possible activation energy involved in switching over from tetrahedral to octahedral bonding, we would not expect that temperature would play an important role in the kinetics of such pressure-induced tetrahedral to octahedral conversions. This may explain the fact that major pressure induced changes in glass structure occur readily and reversibly at room temperature [Hemley et al., 1986; Willams and Jeanloz, 1987].

(5) The proposed mechanism for coordination changes in melts and glasses may also aid understanding of observed crystal-glass transitions. For example, certain high pressure crystalline phases yield glasses on pressure-quenching [e.g., Liu and Ringwood, 1975; Liu, 1979]. It may be that the high pressure phases can be described in terms of networks of silicate tetrahedra to which the phases displacively relax on release of pressure. The quenchability of stishovite, however, which can be described in terms of such a network (Figure 2a), indicates that such structures are not inevitably unquenchable. Similarly, it could be that transformations of the sort that we propose play a role in the conversion of crystals to glass under pressure at room temperature [e.g., Hemiey, 1987; Williams and Jeanloz, 1987]. In such cases, we would propose that conversion to the stable, high pressure crystalline phase is inhibited by the need for reconstructive transformations; conversion to metastable high pressure glass, however, is not difficult under these low temperature conditions because it can be achieved merely by distortions of the low pressure tetrahedral framework. Likewise, glass formation may be favored in certain shock wave experiments either at high pressures or upon unloading from a high pressure crystalline structure.

Acknowledgments. We thank J. Bass, G. E. Brown, Jr., R. Jeanloz, and $Q$. Williams for suggestions that helped us to develop the ideas presented in this paper, and R. Hemley, J. Holloway, and C. Meade for comments and reviews. We also thank $\mathrm{R}$. Myers for assistance with the computer-generated graphics. This work was supported by NSF Grants EAR-8407784 and EAR-8618545. Caltech Division of Geological and Planetary Sciences Contribution 4471.

\section{References}

Arndt, J., Densification of glasses of the system $\mathrm{TiO}_{2}-\mathrm{SiO}_{2}$ by very high static pressures, Phys. Chem. Glasses, 24, 104-110, 1983.

Arndt, J., and D. Stoffler. Anomalous changes in some properties of silica glass densified at very high pressures. Phys. Chem. Glasses, 10, 117-124, 1969.

Bloss, F. D., Crystallography and Crystal Chemistry, 545 pp., Holt, Rinehart and Winston, Inc., 1971.

Boettcher, A. L., Q. Guo, S. Bohlen, and B. Hanson, Melting in feldspar-bearing systems to high pressures and the structure of aluminosilicate liquids, Geology, 12, 202-204, 1984.

Boslough, M. B., S. M. Rigden, and T. J. Ahrens, Hugoniot equations of state of anorthite glass and lunar anorthosite, Geophys. J. Roy. Astr. Soc., 84, 455-473, 1986.

Grimsditch, M., Polymorphism in amorphous $\mathrm{SiO}_{2}$, Phys. Rev. Lett., 52, 2379-2381, 1984.

Hemley, R. J., Pressure dependence of Raman spectra of $\mathrm{SiO}_{2}$ polymorphs: $\alpha$-quartz, coesite, and stishovite, in High Pressure Research in Mineral Physics, edited by M. H. Manghnani and Y. Syono, in press, 1987.

Hemley, R. J., H. K. Mao, P. M. Bell, and B. O. Mysen, Raman spectroscopy of $\mathrm{SiO}_{2}$ glass at high pressure, Phys. Rev. Lett., 57, 747-750, 1986.

Liu, L.-G., The system enstatite-wollastonite at high pressures and temperatures, with emphasis on diopside, Phys. Earth Planet. Int., 19, P15-P18, 1979.

Liu, L.-G., and A. E. Ringwood, Synthesis of a perovskite-type polymorph of $\mathrm{CaSiO}_{3}$, Earth Planet. Sci. Lett., 28, 209-211, 1975.

Mozzi, R. L., and B. E. Warren, The structure of vitreous silica, J. Appl. Crystallogr., 2, 164-172, 1969.

Navrotsky, A., K. L. Geisinger, P. McMillan, and G. V. Gibbs, The tetrahedral framework in glasses and melts - Inferences from molecular orbital calculations and implications for structure, thermodynamics, and physical properties, Phys. Chem. Minerals, 11 , 284-298, 1985.

Ohtani, E., F. Taulelle, and C. A. Angell, $\mathrm{Al}^{3+}$ coordination changes in liquid aluminosilicates under pressure, Nature, 314, 78-81, 1985.

Okuno, M., and F. Marumo. The structures of anorthite and albite melts. Mineral. J. (Japan), 11, 180-196, 1982.

Rigden, S. M., T. J. Ahrens, and E. M. Stolper, Densities of liquid silicates at high pressures, Science, 226, 1071-1074, 1984.

Rigden, S. M., T. J. Ahrens, and E. M. Stolper, Shock compression of molten silicate: Results for a model basaltic composition, J. Geophys. Res., in press, 1987.

Schmitt, D. R., I. Applications of double exposure holography to the measurement of in situ stress and the elastic moduli of rocks from boreholes. II. Shock temperature measurements in fused quartz and crystalline $\mathrm{NaCl}$ to $35 \mathrm{GPa}$, Ph.D. thesis, $178 \mathrm{pp}$., California Institute of Technology, May 1987.

Taylor, M., and G. E. Brown, Jr., Structure of mineral glasses - I. The feldspar glasses $\mathrm{NaAlSi}_{3} \mathrm{O}_{8}, \mathrm{KAlSi}_{3} \mathrm{O}_{8}, \mathrm{CaAl}_{2} \mathrm{Si}_{2} \mathrm{O}_{8}$, Geochim. Cosmochim. Acta, 43, 61-75, 1979.

Wackerle, J., Shock-wave compression of quartz, J. Appl. Phys., 33, 922-937, 1962.

Williams, Q., and R. Jeanloz, High pressure coordination changes in silicates: Is glassy octahedral silicon unquenchable? (abstract), Final Program and Abstracts (Materials Research Society, 1987 Spring Meeting), 312, 1987.

Edward M. Stolper and Thomas J. Ahrens, Division of Geological and Planetary Sciences, California Institute of Technology, Pasadena, CA 91125

(Received May 15, 1987;

accepted September 25, 1987.) 